\title{
An Adaptive Pointing and Correction Algorithm Using Genetic Approach
}

\author{
Jung Jae Jo and Young Chul Kim \\ Dept. Electronics and Computer Engineering, \\ Chonnam National University, Korea \\ mpisto999@naver.com,yckim@chonnam.ac.kr
}

\begin{abstract}
In this paper, we propose a new user-adaptive pointing and correction algorithm applied in the field of smart sensing. The error from the accelerometer sensor's output must be carefully managed as the sensor is more sensitive to data change compared to that of the gyroscope sensor. Thus, we minimize noise by applying the Kalman filtering to data for each axis from the accelerometer. In addition, we can also obtain effect compensating hand tremor by applying the Kalman filter to the data variation for $x$ and $y$. In this study, we extract data through the Quaternion mapping process on data from the accelerometer and gyroscope. In turn, we can obtain a tilt compensation by applying the compensation algorithm with acceleration of the gravity of the extracted data. Moreover, in order to correct the inaccuracy on smart sensors due to the rapid movement of a device, we propose and integrate a genetic approach.
\end{abstract}

Keywords: Quaternion, Rodrigues, Mapping, Pointing, Correction, Tilt compensation, Kalman filter, Noise, Hand tremor, Bluetooth SPP, Socket program

\section{Introduction}

Motion recognition technology became available beginning with the growth of MEMS (Micro Electro Mechanical System) sensor technology. The rapid progress is continually developing in the field of hardware, including sensors, while an explosive growth of the smartphone market is taking place with related applications, service, social commerce and cloud computing. In addition, it has been accelerating due to the advent of the Tablet PC and interactive game consoles [1]. In particular, the motion recognition interface using MEMS sensors has been highlighted due to the expansion of these MEMS sensor markets and applications. In this paper, a pointing and correction algorithm has been implemented using the MEMS sensor mounted accelerometer and gyroscope sensors.

The existing pointing algorithm requires the geomagnetic sensor as well as the accelerometer and gyroscope sensors [2]. In this study, we implement the pointing and correction algorithm using only the accelerometer and gyroscope sensors without using the geomagnetic sensor that can detect the azimuth. This pointing and correction algorithm has the advantages of having a relatively low cost and power consumption by not using the geomagnetic sensor, but this algorithm is frequently affected by the acceleration of gravity.

The smooth movement of a device mounted motion sensor stably changes the variation of the accelerometer sensor data including the acceleration of gravity. However, the sudden movement of a device mounted motion sensor unstably changes 
the variation of the accelerometer sensor data including the acceleration of gravity. To compensate for this sudden movement, an additional correction algorithm is necessary to get reliable results. Thus we propose the adaptive pointing and correction algorithm by using the genetic algorithm according to the characteristics of the user. A genetic algorithm has the advantage that can get the near-optimal and efficient search in a complex combination of problems. In addition, by setting the additional criteria value about the tilt compensation algorithm using the acceleration of gravity, results for the rapid movement of the device can be optimized.

Section 2 briefly describes existing pointing and correction algorithms as well as connectivity for the adaptive pointing and correction algorithm using the genetic algorithm proposed in this paper. Section 3 covers how to solve problems when a device mounted motion sensor is moved rapidly vertically or horizontally. In addition, we analyze and compare the performance of our proposed adaptive algorithm to those of the existing methods. The final section presents a discussion of this study and future research directions.

\section{Proposed Algorithm}

In this paper, we propose the process of the pointing and correction algorithm as shown in Figure 1. The proposed and developed pointing and correction process can be largely divided into three parts. The first part is a pointing process using the Quaternion mapping process, the second is for tilt compensation, and the third is for correction using the Kalman filter [3, 4].

The mapping process of Quaternion or Rodrigues can be used as the pointing algorithm using the fusion of an accelerometer and gyroscope sensor. Although Quaternion and Rodrigues mapping share the accelerometer and gyroscope sensor data, these two mapping processes have differences in principle. The Quaternion mapping uses axis-transformation to solve the Gimbal lock problem while the Rodrigues mapping uses vector-transformation with the fixed axis. Thus, when a device is tilted, Quaternion mapping cannot reflect the tilt value while the Rodrigues mapping can. Consequently, we confirmed that the Quaternion mapping is more efficient than the Rodrigues mapping in terms of the performance, computation rate and, compensation effect.

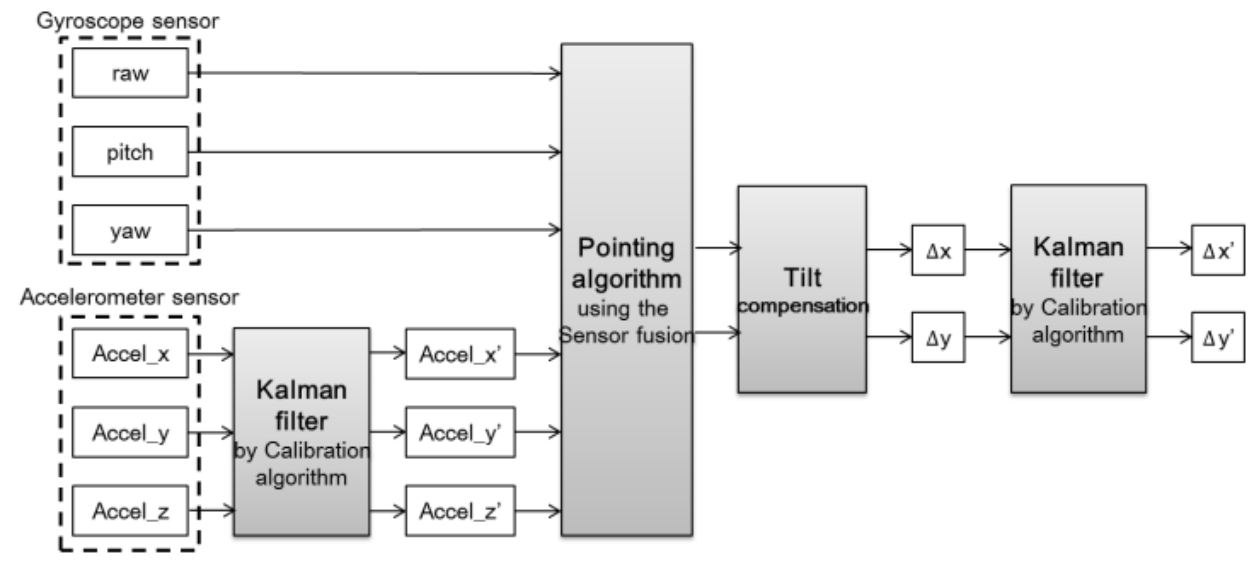

Figure 1. The proposed pointing and correction process 
The additional tilt compensation algorithm was applied to compensate the tilt information about the Quaternion mapping. We can get the tilt information from the accelerometer sensor because this sensor includes the acceleration of gravity for each axis [5]. The variation of the accelerometer sensor has the periodicity. Based on this periodicity, we can confirm the similarity between the unit value of the $\mathrm{z}$-axis and cosine function by calculating the unit value for each axis. So, we can obtain the tilt angle by using the unit value of the z-axis. The unit value of the z-axis is important data to generate the initial population related to the genetic algorithm.

The range of the tilt angle is from 0 to $2 \pi$. If a device is rotated to the left, the information of the tilt angle is matched. However, if it is rotated to the right, the information of the tilt angle is reversed. To solve this problem, the tilt angle is induced as shown in Figure 2 [6].

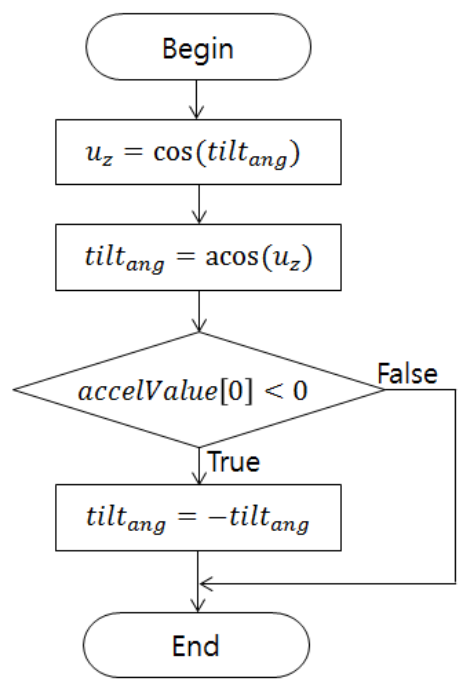

Figure 2. The proposed tilt compensation algorithm

If a device makes a sudden movement, the accelerometer sensor may show incorrect data. In addition, the conditional statement can be added to complement the tilt compensation algorithm. This part of the conditional statement is to implement the adaptive pointing and correction algorithm using a genetic algorithm.

The $\mathrm{x}$ and $\mathrm{y}$ variation of display is sensitive due to the change of the accelerometer sensor. For this reason, we apply the Kalman filter as the calibration algorithm for minimization of sensor noise. Also, we can get the compensation effect to alleviate hand tremor by applying the Kalman filter after the tilt compensation algorithm.

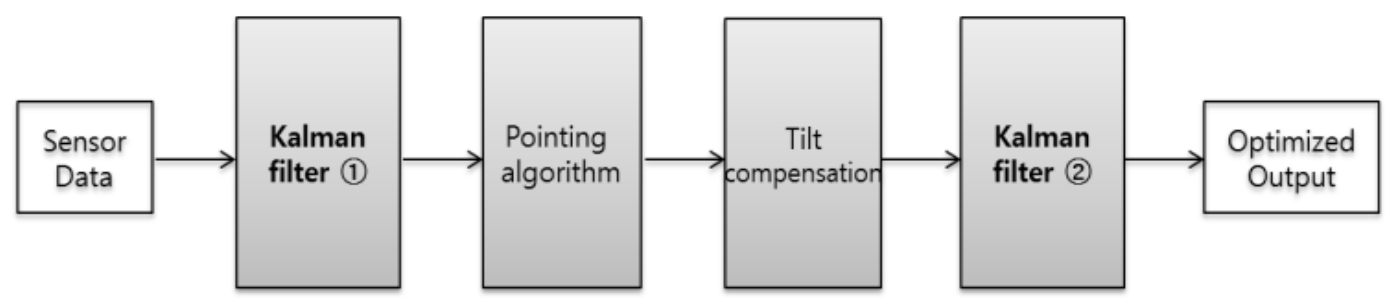

Figure 3. Implementation of the Kalman filter 
These Kalman filters are implemented based on the measurement updating algorithm to update the new optimal value using the output value of the system and the new input value [7]. In this paper, the unit value of the z-axis is used to generate the initial population for the genetic algorithm. The generation of initial population plays important role in our genetic algorithm.

Additional correction algorithm is needed to minimize the noise of sensor (1) and reduction of the hand tremor(2). In this paper, we have implemented the correction algorithm using the Kalman filter shown in Figure 6. This implemented filter is based on the measurement update algorithm to update the new optimal value using the output value of the system and the new input value $[8,9]$.

The optimal value can be induced based on the importance of the data replaced by the recursive data processing and the standard deviation corresponding to the basic theory of Kalman filter shown in the following equations.

$$
\begin{aligned}
& \text { Estimate variance }:(s d)^{2}=\frac{\left(s d_{1}\right)^{2}+\left(s d_{2}\right)^{2}}{\left(s d_{1}\right)^{2}\left(s d_{2}\right)^{2}} \\
& \text { Cf) } s d: \text { process noise } \\
& s d_{1}: \text { measurement noise for } \mathrm{x}(\mathrm{t}-1) \\
& s d_{2}: \text { measurement noise for } \mathrm{x}(\mathrm{t}) \\
& \text { Optimized state variables : } X=X_{1}+\left(X_{2}-X_{1}\right)\left[\frac{(s d)^{2}}{(s d)^{2}+\left(s d_{2}\right)^{2}}\right] \\
& \text { Cf) } X_{1}: \text { The output value of the system, } X(t-1) \\
& X_{2}: \text { The new input value, } X_{n}
\end{aligned}
$$

$\operatorname{sd}_{1}$ and $\operatorname{sd}_{2}$ is continuously updated using the standard deviation. Based on these two values, Process noise is also updated. New input value means currently updated data and represents the previous optimum value.

In this paper, we apply the tilt compensation algorithm using the accelerometer sensor. Without using the tilt compensation algorithm, the rapid movement of a device has no effect on the variation of coordinates on display. Meanwhile, we can observe that the sudden movement of a device results in a negative effect when the tilt compensation algorithm is used. There is a close correlation between the unit value of the $\mathrm{z}$-axis and cosine function in the process of inducing the tilt angle. It is also confirmed that the change of tilt is large when tilt angle is close to 90 degrees while closing to 0 degrees results in a small change of tilt. This is the basis of minimal distortion in the $\mathrm{x}$ and $\mathrm{y}$ variation when the tilt angle of a device is close to 90 degrees with the sudden movement of the device. The condition can be applied by using the variation characteristics of the tilt angle. This conditional statement determines whether to use for the tilt compensation algorithm based on specific value or not.

The unit value of the z-axis, which is generated using the sensor data of an accelerometer compensated through the Kalman filter, is replaced by the index of the applied genetic algorithm. The index, which is the initial population, is the object in the environment of rapid movement. This object is stored temporarily as a permutation, and finally settles to an optimal value through the genetic algorithm. The optimized result is the object with the maximum suitability resulted from the applied share-based suitability method [8]. In this paper, the process to derive the optimized tilt reference value is integrated into the general pointing and correction algorithm, as shown in Figure 4. 
The creating conditions of the initial population, which are obtained from the data value of the accelerometer sensor caused by the rapid movement of a device, are converted into unit value of the z-axis. The suitability to any solution is the index value referencing when selecting a right one from solution group. The suitability also reflects quality of the solution. An adjacent pair of solutions is selected as a group based on the initial population generated in function of size of the solution group. The selected group passes through a mixed exchanging method as well as a transforming process with other group of solutions based on the predefined probability in variation [9]. During the described genetic computation, a new population is added to the permutation, and then the suitability to a solution is calculated. This process is repeated until the terminating condition is satisfied. In this paper, we use the share-based method to evaluate the suitability [10]. The object with high suitability is finally selected as a reference value after satisfying terminating condition.

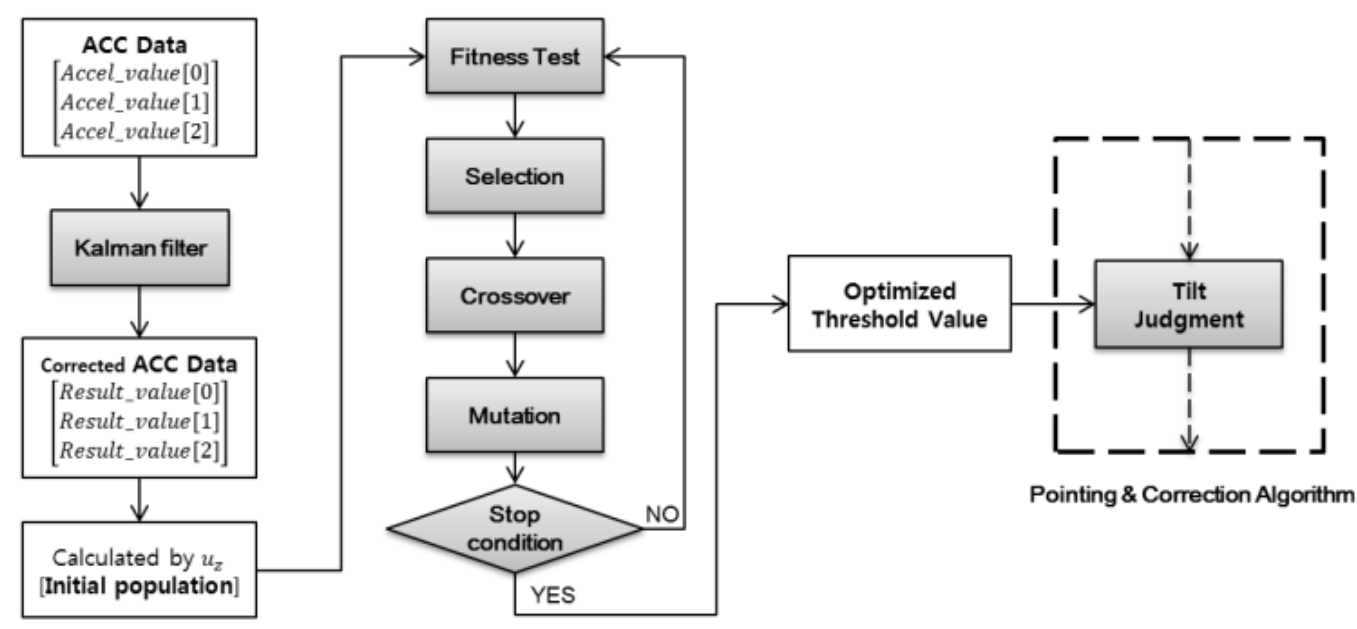

Figure 4. The process of the proposed adaptive pointing and correction algorithm

The adjacent objects from the generated population are grouped and exchanged. A mixed crossover method rather than general distributed crossover techniques is used because the element of the object is not a bit, but a real number $[10,11]$. The searching coefficient in this process can be defined by the user, and represents the positive value. In addition, the process of permutation is used to change the index of elements and other random elements of the index digit using the permutations of the value. The more suitable objects can be obtained through crossbreeding with other groups as generations repeat by using the method of variation probability $[12,13]$.

\section{Analysis and Performance}

The operation rate and variation is important factors in the pointing and correction algorithm. In this paper, we analyzed and compared the operation rate on the transmitter, and $\mathrm{x}$, y variation applied to each mapping process in the case of a fixed device. These comparisons are shown in Table 1 and Figure 5.

In the fixed device, the performance of the Quaternion mapping is superior to that of the Rodrigues mapping because the output of $x$ and $y$ variation is closed zero value. 
Table 1. The comparison of average operation rate in case of a fixed device.

\begin{tabular}{|c|c|c|}
\hline The number of loop & Quaternion mapping & Rodrigues rotation mapping \\
\hline $1-100$ & $382,125 \mathrm{~ns}$ & $411,118 \mathrm{~ns}$ \\
\hline $101-200$ & $300,005 \mathrm{~ns}$ & $442,801 \mathrm{~ns}$ \\
\hline $201-300$ & $258,423 \mathrm{~ns}$ & $423,145 \mathrm{~ns}$ \\
\hline $301-400$ & $196,343 \mathrm{~ns}$ & $406,271 \mathrm{~ns}$ \\
\hline $401-500$ & $225,620 \mathrm{~ns}$ & $435,145 \mathrm{~ns}$ \\
\hline $501-600$ & $227,691 \mathrm{~ns}$ & $680,828 \mathrm{~ns}$ \\
\hline $601-700$ & $279,900 \mathrm{~ns}$ & $919,970 \mathrm{~ns}$ \\
\hline $701-800$ & $480,468 \mathrm{~ns}$ & $838,399 \mathrm{~ns}$ \\
\hline $801-900$ & $533,706 \mathrm{~ns}$ & $919,483 \mathrm{~ns}$ \\
\hline $901-1000$ & $374,154 \mathrm{~ns}$ & $646,239 \mathrm{~ns}$ \\
\hline Average & $325,844 \mathrm{~ns}$ & $612,340 \mathrm{~ns}$ \\
\hline
\end{tabular}

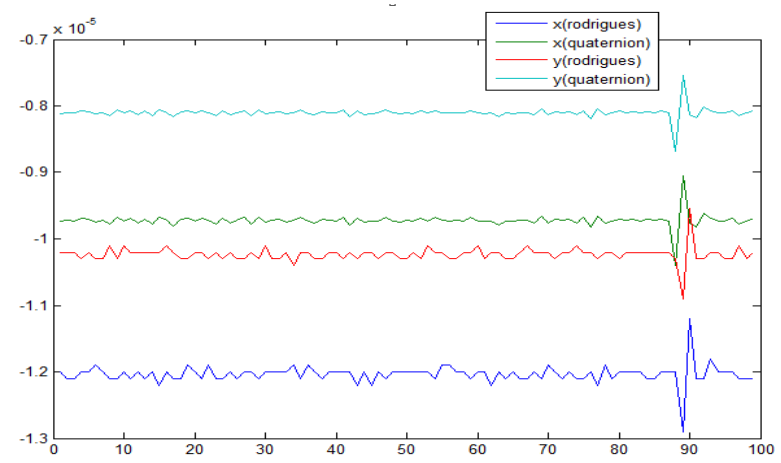

Figure 1. The comparison of $x, y$ variation applied to each mapping process in the case of a fixed device

The comparison results of the before and after applying the Kalman filter in case of a fixed device and a moving device are shown in Figure 6 and 7, respectively. It shows the minimized sensor noise in our proposed approach.

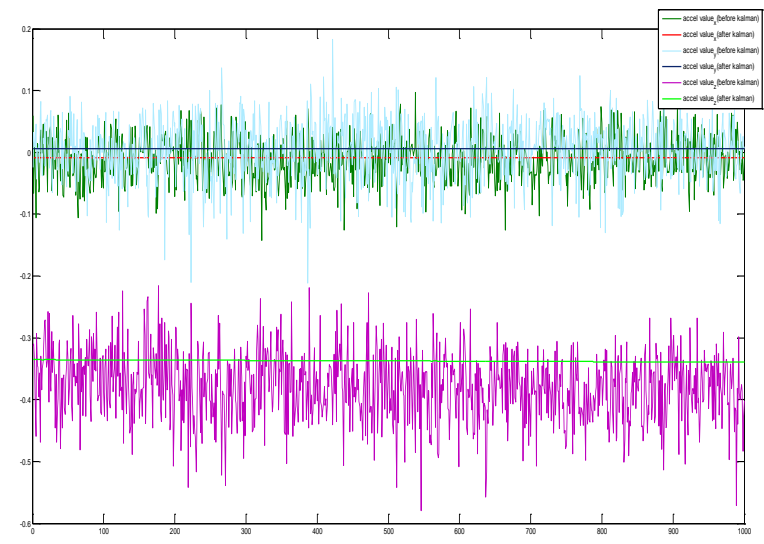

Figure 6. Sensor data of the before and after applying Kalman filter in the case of a fixed device 


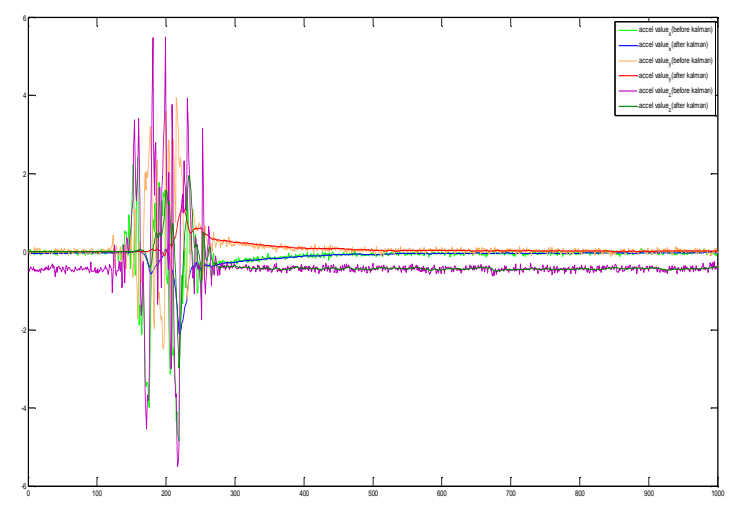

Figure 2. Sensor data of the before and after applying Kalman filter in the case of a moving device

The comparison results in $\mathrm{x}$ and $\mathrm{y}$ variation before and after applying the Kalman filter are shown Figure 8 and. 9, respectively. We also can solve the problem of hand tremor by applying an additional Kalman filter on $\mathrm{x}$, $\mathrm{y}$ variation.

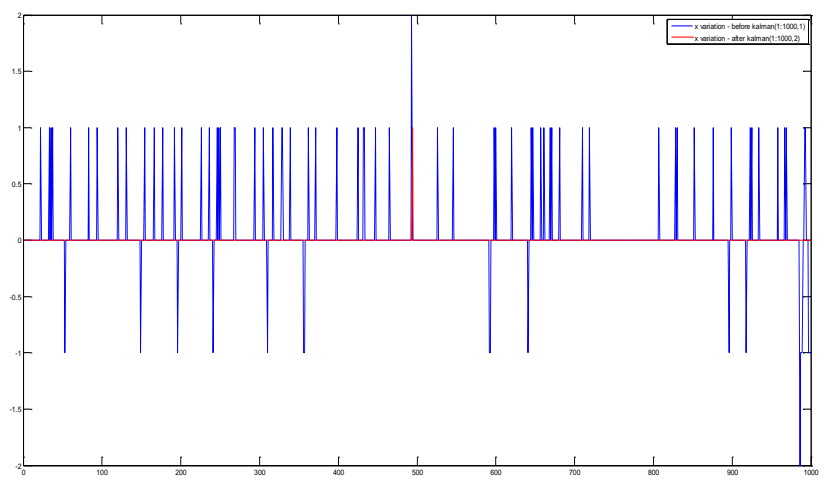

Figure 3. $X$ variation before and after applying Kalman filter

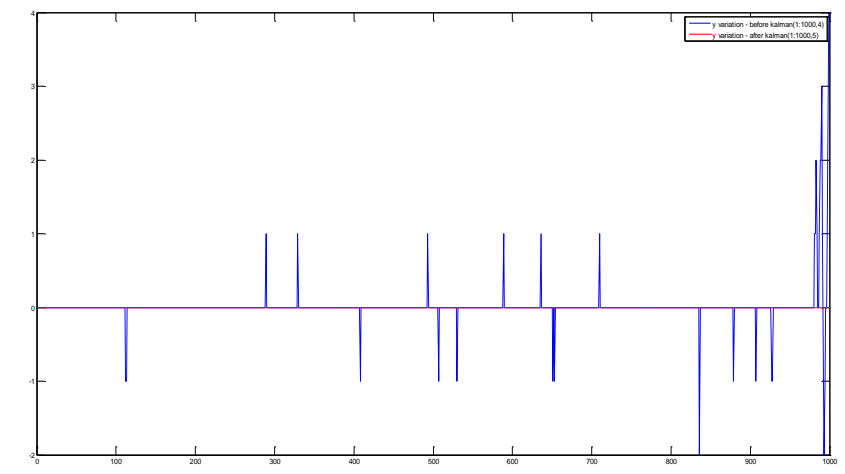

Figure 4. Y variation before and after applying Kalman filter 
Bluetooth SPP (Serial Port Profile) uses the RFCOMM protocol and is configured with a serial cable connection between the Bluetooth host and the device. By setting the Bluetooth SPP UUID, the host and the device are connected with the Bluetooth communication. The development environment for transceivers based on Bluetooth communication is shown in Figure 10.

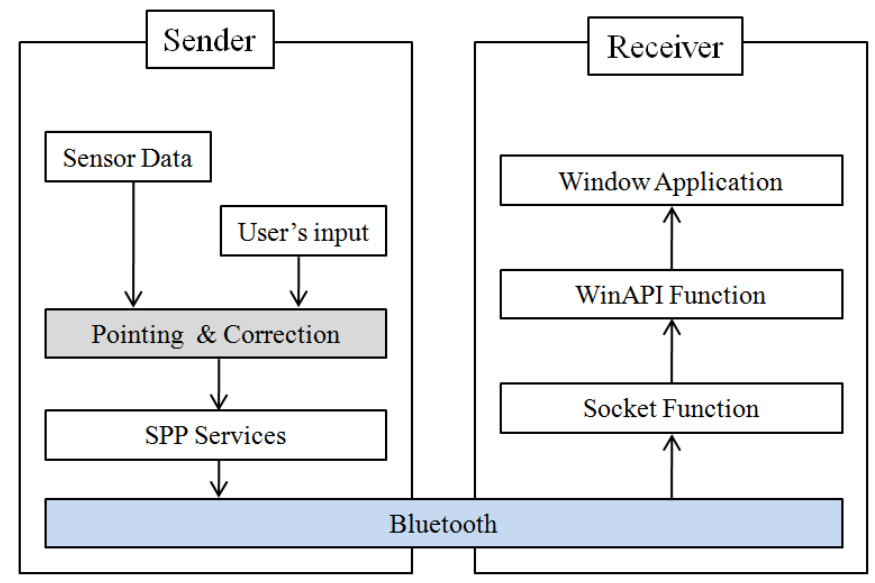

\section{Figure 5. Development environment of transceivers based on Bluetooth communication}

Communication of our server-client model is configured by implementing a Bluetooth socket program. The host and the device create a socket, and then the host creates a communication port. Until the host receives a connection request from the device, each waits at the generated port. Then, the device transmits data after the host receives a connection request and authorized connection.

\section{Conclusions}

In this study, the user-adaptive pointing and correction algorithm is proposed. It is implemented by applying a genetic approach to the conventional pointing and correction algorithm using smart sensors such as an accelerometer and a gyroscope. Also, the Kalman filter for correction is integrated to minimize the sensor noise while the performance degradation of the sensor in the case of rapid movement is effectively improved by our proposed method.

Our adaptive method includes the shared-based suitability test, the mixed crossover technique, and the method of probability of variation. The algorithm derives the nearest value as a reference depending on a user. In addition, the computational process can be minimized by utilizing the corrected data obtained by Kalman filtering as an initial population in our proposed genetic approach.

The use of interface, interconnecting human gestures with smart devices, will be increased in the field of smart sensing. Our future will be focused on developing a new interfacing system extracting user experience by introducing the NUI (Natural User Interface) beyond the use of conventional smart sensors such as accelerometers and gyroscopes, where The development of such a method as approaching an analog environment, which is most appropriate for interfacing human to devices, will be one of the key issues in the research. 


\section{Acknowledgements}

This research was financially supported by the Ministry of Education, Science Technology (MEST) and National Research Foundation of Korea (NRF) through the Human Resource Training Project for Regional Innovation (No. 2012-04A0301912010100).

\section{References}

[1] J. -Y. Song, "Smart TV and mobile devices, the paradigm of change and the development of the Smart Media", KT Institute of Economic and Business Administration, (2011) December 26.

[2] A. Kim and M. F. Golnaraghi, "Initial Calibration of an Inertial Measurement Unit Using an Optical Position Tracking System", Proceedings of Position Location and Navigation Symposium, IEEE, (2004), pp. 96-101.

[3] J.-R Kim and I.-B. Jeong, "Multiple Dimension User Motion Detection System based on Wireless Sensors", J. Korean Institute of Information and Communication Engineering, vol. 15, no. 3, (2011), pp. 700-712.

[4] Kuipers J. B, “Quaternions \& Rotation Sequences”, Coral Press, (2000) Sep. 1-10, pp. 127-143, Grand Rapids, USA.

[5] T. Ozyagcilar, "Implementing a Tilt-Compensated eCompass using Accelerometer and Magnetometer Sensors", Freescale Semiconductor, AN 4248, Rev. 3, (2012) Jan.

[6] P. Kumar, J. Verma and S. Prased, "Hand Data Glove: A Wearable Real-Time Device for Human Computer Interaction", J. International Journal of Advanced Science and Technology, vol. 43, no. 2 , (2012) June, pp. 15-26.

[7] G. Welch and G. Bishop, “An Introduction to the Kalman Filter”, UNC-Chapel Hill, TR 95-041, (2006) July 24, USA.

[8] B.-K. Sohn and K.-M. Lee, "A Coordinated Collaboration Method of Multiagent Systems based on Genetic Algorithms”, J. Korean Institute of Intelligent Systems, vol. 14, no. 2, (2004) Mar. 28, pp. 156-163.

[9] H. Faris and A. F. Sheta, "Identification of the Tennessee Eastman Chemical Process Reactor Using Genetic Programming", J. International Journal of Advanced Science and Technology, vol. 50, no. 11, (2013), pp. 121-140.

[10] T. Yalcinoz, H Altun and M. Uzam, "Economic Dispatch Solution Using A Genetic Algorithm Based on Arithmetic Crossover", Proceedings of Power Tech Conference 10-13 ${ }^{\text {th }}$, IEEE, (2001) September 10-13, Porto, Portugal.

[11] A. Brezulianu, M. Fira and L. Fira, "A Genetic Algorithm Approach for a Constrained Employee Scheduling Problem as Applied to Employees at Mall Type Shops", J. International Journal of Advanced Science and Technology, vol. 14, no. 1, (2010), pp. 1-14.

[12] J. Smith and T. C. Fogarty, "Self Adaptation of Mutation Rates in a Steady State Genetic Algorithm”, Proceedings of IEEE International conference, (1996) May 20-22, pp. 318-323, Nagoya, Japan.

[13] N. Birla and A. Swarup, "Performance of Preview Control based on Evolutionary Algorithms", J. International Journal of Advanced Science and Technology, vol. 38, no. 4, (2012), pp. 37-52.

\section{Authors}

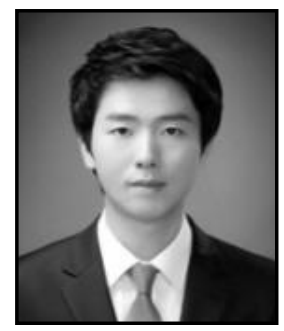

\section{Jung Jae Jo}

He received his BS in electronics engineering from Chonnam National University, Korea. He is now pursuing his Master of Science in Electron ic Engineering at Chonnam National University. His research area interes ts include information systems applications, design tools and techniques of user interface. 


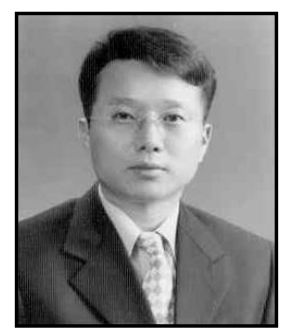

\section{Young Chul Kim}

He received received his $\mathrm{PhD}$ from Michigan State University, USA, the MS from the University of Detroit, USA, and BS in electronics engineering from Hanyang University, Korea. In 1993, he joined the Department of Electronics Engineering at Chonnam National University (CNU) where he is currently a professor. From 2000 to 2004, he was a director of IDEC at CNU. From 2004 to 2005, he was a Vice Dean of the College of Engineering in this university. Since 2004, he has become the chief of the LG Innotek R\&D center at CNU. His research interests are SoC design and development using IPs and low power design. 